\title{
Clinical, Neurophysiological and Histopathological correlations in Pure Neural Leprosy
}

\author{
Izabela Jardim Rodrigues Pitta 1, 2, 3, *, Bruno de Siqueira Mietto 4, Aron dos Santos Camilo 3, \\ Sergio Luiz Gomes Antunes 2, Euzenir Nunes Sarno 2, Marcia Rodrigues Jardim 1, 2, 3
}

\begin{abstract}
1 Programa de Pós Graduação em Neurologia (PPGNeuro), Universidade Federal do Estado do Rio de Janeiro (UNIRIO), Rio de Janeiro, RJ, Brazil.

${ }_{2}^{2}$ Instituto Oswaldo Cruz (IOC), Fundação Oswaldo Cruz (Fiocruz), Rio de Janeiro, RJ, Brazil.

${ }^{3}$ Universidade do Estado do Rio de Janeiro, UERJ, Rio de Janeiro, RJ, Brazil.

${ }^{4}$ Instituto de Ciências Biológicas, Universidade Federal de Juiz de Fora, Juiz de Fora, MG, Brasil.
\end{abstract}

*Corresponding author: Izabela Jardim Rodrigues Pitta Instituto Oswaldo Cruz (IOC), Fundação Oswaldo Cruz (Fiocruz), Av Brasil 4365, Rio de Janeiro, CEP21041-361. Phone: +55(21)2562-1594. E-mail: dra.izabela.pitta@gmail.com.

Research Ethics Committee Approval: Approved by the Research Ethics Committee of the Fundação Oswaldo Cruz (1.787.835).

Received on: Aug16, 2021. Accepted on: Aug 26, 2021. Available online: Nov 05, 2021.

\begin{abstract}
Although neuropathy remains one of the most problematic issues faced by leprosy patients, the evolving process of its findings continues a challenge particularly in pure neural leprosy (PNL). We evaluated neurological examination, nerve conduction studies and histopathological data of patients with PNL and ulnar neuropathy. Patients with longer duration of symptoms had reduction in the motor conduction velocities and patients with fibrosis in the biopsy had axonal damage in the nerve conduction studies. This suggests that focal demyelination may be present in leprosy patients at the moment of the diagnosis and be related to the duration of the neuropathy.
\end{abstract}

Keywords: Leprosy; Neuropathy; Ulnar Neuropathy; Nerve Conduction Studies.

\section{Introduction}

Leprosy neuropathy is a chronic infectious disorder of the peripheral nerve system, caused by the intracellular pathogen Mycobacterium leprae entry and persistence in the nerve microenvironment

$$
\text { [1]. Leprosy }
$$

neuropathy is characterized by extensive nerve damage, which includes demyelination and axon loss, leading to impairment of nerve function for patients. Leprosy is usually diagnosed in two major disease spectrum, accordingly to their bacterial load and 
immunological responses, as described by Ridley-Jopling [2]. However, some of leprosy neuropathy patients manifest a quiescent, silent form of the neural disease, called pure neural leprosy (PNL), where patients have no skin lesions and only manifest neurological clinical symptoms [3].

Because of the absence of skin lesions, the diagnosis of PNL is sometimes challenging and, therefore, nerve conduction studies (NCS) are an important tool to evaluate the presence and extension and degree of nerve impairment in clinics [4]. NCS are considered an extension of the clinical examination as they can infer the underlying pathophysiology of the neuropathy. They are also important to indicate whether it is caused by axonal degeneration, demyelination or both [5]. Many researchers have shown the presence of axonal patterns in leprosy neuropathy nerve conduction studies (NCS) $[3,4,6]$. However, recently it has been more frequently described by our group and others, that demyelinating patterns could occurs in early stages of the disease [7-8].

Leprosy neuropathy can be developed in any nerve trunk of the peripheral nervous system, but the ulnar nerve is one of the most affected ones during diseases [3,9-10]. Therefore, taking into consideration the lack of available neurophysiological data for leprosy neuropathy patients with accompanied histopathological examination of the nerve biopsies, the objective of the present study was to evaluate the nerve conduction studies in a group of PNL undergoing ulnar nerve biopsy.

\section{Methodology}

The present study was carried out at the Souza Araujo Outpatient Clinic, leprosy reference center in Rio de Janeiro state, Brazil, and was approved by the Research Ethics Committee of the institution. Patients diagnosed with PNL through the biopsy dorsal cutaneous branch of the ulnar (DCUB) nerve were selected. Patients with comorbidities related to peripheral neuropathies such as diabetes mellitus, rheumatic diseases, alcoholism, B12 hypovitaminosis, the HIV virus, or viral hepatitis were excluded from the study.

Patients were first assessed by dermatologists to excluded evidence of skin disease and then proceeded to slit smears analysis to determine the systemic bacillary load. The patients were then evaluated by a neurologist and submitted to a neurological examination focused on peripheral neuropathy as was described by Jardim et al. [3].

Patients with clinically-suspected PNL were submitted to NCS, which were performed using the same Nihon- 
Koden MEB-9400 apparatus in accordance with standard procedures. All patients had their median, ulnar, radial, sural and superficial peroneal sensory nerves evaluated. They also had the median, ulnar, peroneal and tibial motor nerves evaluated. The NCS were conducted as described by Vital et al. [4].

Nerve sample biopsies of the DCBU were obtained for use in diagnostic histological procedure. Nerve segments were divided into small fragments: one for the histopathological routine studies and the another to identify $M$. leprae DNA by q-PCR procedure. Briefly, nerve segments were fixed in $2.5 \%$ glutaraldehyde diluted in $0.1 \mathrm{M}$ cacocylate buffer and post-fixed in $1 \%$ osmium tetroxide. The segments, were then dehydrated in acetone and embedded in Epon resin. Semithin cross sections of the nerve were obtained using a Reichert ultramicrotome and sections were stained for toluidine blue for nerve architecture analysis. The criteria used in the histopathological diagnoses were those described by Antunes et al. [11].

The data analysis was performed using the Fisher's exact test for categorical variables and the MannWhitney test was performed for the numerical variables. The Statistical Package for the Social Sciences (SPSS) 16 was used for data analysis.

\section{Results}

Fifteen patients diagnosed with PNL through the DCUB biopsy were included. The mean age of the patients was 46.3 years and the mean duration of symptoms was of 45.2 months. 53.3\% (7 patients) were male and $46.7 \%$ (7 patients) were female.

At neurological examination, $40 \%$ of the patients (6 patients) had neuropathic pain at the diagnosis. $66.7 \%$ of the sample (10 patients) had impairment in pain and thermal sensitivity, $46.7 \%$ (7 patients) had impairment in tactile sensitivity and $60 \%$ (9 patients) had weakness in the ulnar nerve territory. $33.3 \%$ of our sample (5 patients) had enlargement of the ulnar nerve.

The NCS studies data are summarized in table 1 . All of the patients showed abnormalities in their NCS. Mononeuropathy and multiplex mononeuropathy were the most common extension pattern, as each had $46.7 \%$ of the patients. When evaluating the neurophysiological injury pattern, demyelinating features were the most commonly observed, as they were present in $86.6 \%$ of the patients, alone or in combination with axonal features.

The morphological appearance of the nerves was examined to substantiate our nerve conduction findings (Figure 1). Five patients (33.3\%) had an 
inflammatory infiltrate in the biopsied nerve and six patients $(40 \%)$ had fibrosis. Only two patients $(13.3 \%)$ had acid fast bacilli found in the sample. The sample revealed decrease on the number of myelinated fibers in thirteen patients $(86.7 \%)$.

Table 1. Nerve Conduction Studies data.

\begin{tabular}{llc}
\hline & Nerve Conduction Studies & Number (\%) \\
\hline Extension of NCS & Mononeuropathy & $7(46.7 \%)$ \\
& Multiplex Mononeuropathy & $7(46.7 \%)$ \\
NCS pattern & Polyneuropathy & $1(6.7 \%)$ \\
& Demyelinating & $8(53.3 \%)$ \\
Ulnar nerve sNCS & Axonal & $2(13.3 \%)$ \\
& Mixed & $5(33.3 \%)$ \\
& Normal & $2(13.3 \%)$ \\
& Reduced SNAP amplitude & $5(33.3 \%)$ \\
& No conduction & $8(53.3 \%)$ \\
& Conduction Block & $4(26.7 \%)$ \\
& Reduction in motor conduction velocity & $12(80 \%)$ \\
& Reduced CMAP amplitude & $6(40 \%)$ \\
& No conduction & $2(13.3 \%)$ \\
& &
\end{tabular}

Legend: NCS: nerve conduction studies. SNAP: sensory nerve action potential. CMAP: compound muscle action potential. sNCS: Sensory Nerve Conduction Studies. mNCS: Motor Nerve Conduction Studies.

There was no statistically significant association between the duration of symptoms and the clinical or histopathological findings, but there was an association between the longer duration of symptoms and the presence of reduced motor conduction velocity.
The duration of the symptoms and the clinical, neurophysiological and histopathological data are summarized in table 2. 

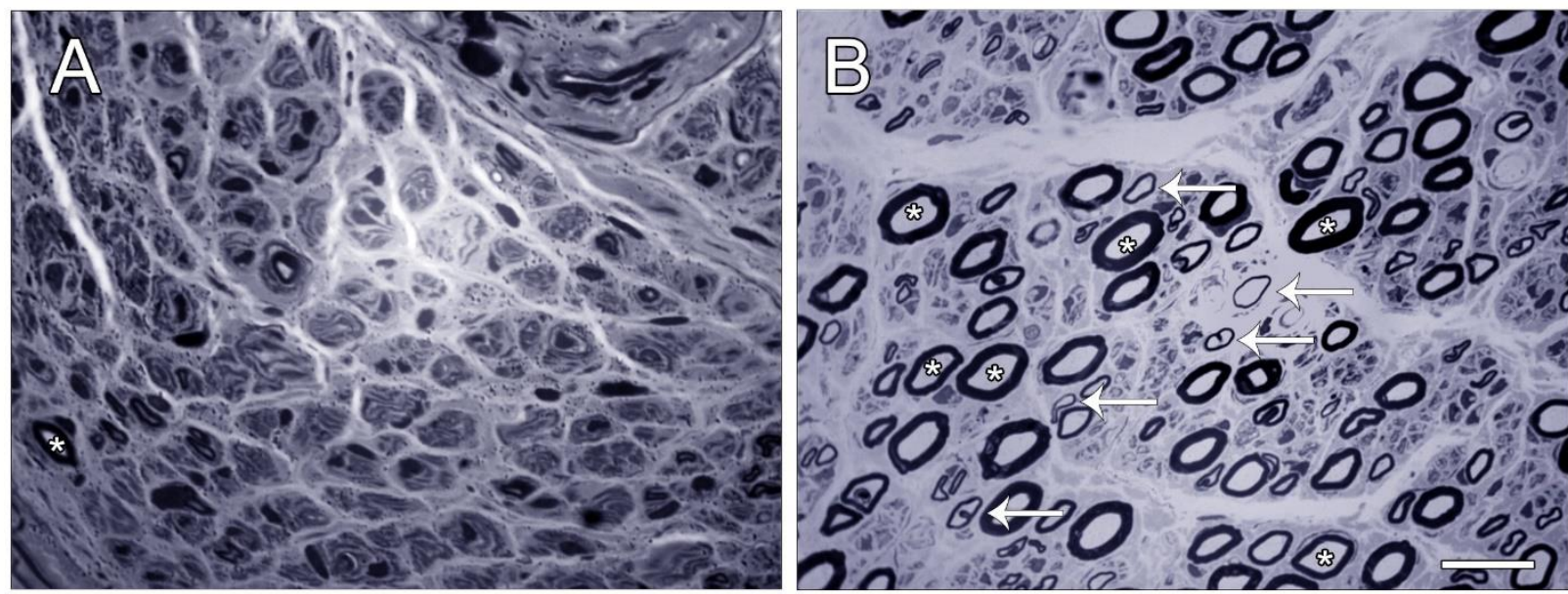

Figure 1: Pure neural leprosy nerve biopsies. Toluidine blue stain. 100x magnification. Semithin section of peripheral nerve from two leprosy patients. Patient A: revealed a decrease in the quantitty of myelinated fibers. The endoneurial compartment is filled with few small myelinated fibers, remaining profiles of unmyelinated fibers and endoneurial capillary vessels. Patient B: Semithin section of peripheral nerve reveals moderate loss of large myelinated fibers and axons with signs of remyelination (arrows). Scale bar $=10 \mu \mathrm{m}$.

Table 2: Association between the duration of symptoms and clinical, neurophysiological and histopathological data at the diagnosis by number of patients and Mann-Whitney $\mathrm{U}$ test $\mathrm{p}$ - value.

\begin{tabular}{lcccc}
\hline Duration of symptoms & Total & $\begin{array}{c}\text { Nerve Thicknening } \\
\mathbf{p}=\mathbf{0 , 8 0 5}\end{array}$ & $\begin{array}{c}\text { Reduced MCV } \\
\mathbf{p = 0 , 0 4 8}\end{array}$ & $\begin{array}{c}\text { Fibrosis } \\
\mathbf{p}=\mathbf{0 , 4 0 5}\end{array}$ \\
\hline $\mathbf{4}$ months & 1 & 1 & 1 & 1 \\
$\mathbf{5}$ months & 2 & 0 & 0 & 1 \\
$\mathbf{8}$ months & 1 & 0 & 1 & 0 \\
$\mathbf{2 4}$ months & 3 & 1 & 1 & 0 \\
$\mathbf{3 0}$ months & 1 & 0 & 1 & 0 \\
$\mathbf{3 6}$ months & 2 & 1 & 2 & 1 \\
$\mathbf{6 0}$ months & 2 & 1 & 2 & 0 \\
120 months & 3 & 1 & 3 & 3 \\
\hline
\end{tabular}

Legend: $\mathrm{MCV}$ : motor conduction velocity.

When we compare the NCS patterns with the histopathological findings there is an association between the presence of fibrosis and the presence of axonal damage in the NCS (Fisher's exact test, $\mathrm{p}=0.041$ ) as showed in table 3 .
There is also an association between the amplitude of the sensory nerve action potential (SNAP) in $\mu \mathrm{V}$ and the reduction on large myelinated fibers observed in the biopsy. The findings of 
nerve biopsy and SNAP amplitudes are summarized in table 4.

There was no statistically

significant correlation between the

presence of AFB or inflammatory infiltrate with any NCS parameter as demonstrated in table 5.

Table 3: Number of patients with fibrosis and nerve conduction studies abnormalities and Fihser's exact test p-value.

\begin{tabular}{lcc}
\hline & Presence of fibrosis & p-value \\
\hline Presence of axonal damage features on NCS & $5(33.3 \%)$ & $\mathbf{0 , 0 4 1}$ \\
Presence of demyelinating features on NCS & $4(26.6 \%)$ & 0,143 \\
\hline
\end{tabular}

Legend: NCS. Nerve Conduction Studies.

Table 4: Association of the amplitude of the sensory nerve action potential and histopathological findings by number of patients and Mann-Whitney $U$ test $p$-value.

\begin{tabular}{lcccc}
\hline $\begin{array}{l}\text { SNAP } \\
\text { amplitude }\end{array}$ & Total & $\begin{array}{c}\text { Fibrosis } \\
\mathrm{p}=\mathbf{0 . 4 4 3}\end{array}$ & $\begin{array}{c}\text { Inflammatory } \\
\text { infiltrate } \\
\mathbf{p = 0 , 2 3 2}\end{array}$ & $\begin{array}{c}\text { Reduction of large } \\
\text { myelinated fibers } \\
\mathbf{p = 0 , 0 1 2}\end{array}$ \\
\hline $\mathbf{0} \boldsymbol{\mu \mathbf { V }}$ & 8 & 4 & 4 & 8 \\
$\mathbf{1 . 3} \boldsymbol{\mu} \mathbf{V}$ & 1 & 0 & 0 & 1 \\
$\mathbf{3 . 2} \boldsymbol{\mu} \mathbf{V}$ & 1 & 1 & 0 & 1 \\
$\mathbf{6} \boldsymbol{\mu} \mathbf{V}$ & 1 & 0 & 0 & 1 \\
$\mathbf{6 . 6} \boldsymbol{\mu V}$ & 1 & 0 & 0 & 0 \\
$\mathbf{7} \boldsymbol{\mu} \mathbf{V}$ & 1 & 0 & 1 & 0 \\
$\mathbf{1 1} \boldsymbol{\mu V}$ & 1 & 1 & 0 & 1 \\
$\mathbf{1 2 . 3} \boldsymbol{\mu} \mathbf{V}$ & 1 & 0 & 0 & 0 \\
\hline
\end{tabular}

Legend: SNAP. Sensory Nerve Action Potential.

Table 5: Number of patients with inflammatory infiltrate and acid-fast bacilli in the nerve biopsy and the neurophisiological abnormalities.

\begin{tabular}{lccc}
\hline & Reduction in MCV & Conduction Block & Reduction in SNAP amplitude \\
\hline Inflammatory & $4(26,6 \%)$ & $2(13.3 \%)$ & $4(26.6 \%)$ \\
Infiltrate & {$[p=1.000]$} & {$[p=0.560]$} & {$[p=1.000]$} \\
\multirow{2}{*}{ AFB } & $2(13.3 \%)$ & $1(6.6 \%)$ & $1(6.6 \%)$ \\
& {$[p=1.000]$} & {$[p=0.476]$} & {$[p=0.371]$}
\end{tabular}

Legend: AFB. Acid-fast bacilli. MCV. motor conduction velocity. SNAP. Sensory Nerve Action Potential. 


\section{Discussion and Conclusion}

Although leprosy is a wellknown skin disease, the characteristic neural damage associated with it continues be poorly understood [10]. In our study, patients with ulnar neuropathy, who were diagnosed with PNL, were evaluated to analyze and compare their neurophysiological, histopathological and neurologic examination findings.

The patients evaluated had a mean duration of symptoms of over 45 months. Despite the long duration of symptoms in our patients, there is a wide range of nerve injury extension at presentation, as $46.7 \%$ presented only with a mononeuropathy and 53.3\% had already a multiplex mononeuropathy or a polyneuropathy. This could indicate that the extension of the neuropathy is also determined by individual factors as it is observed in skin lesions in the classic forms of leprosy [2]. Others authors have already described a long duration of symptoms before the diagnosis [9,12]. PNL demands a high suspicion degree for its diagnostic and this indicates that it is still poorly recognized, even in regions where leprosy is an endemic disease.

One of the difficulties in correlating the pathological process and the neurophysiology in leprosy neuropathy is the fact that the sensory nerves are profoundly affected in most of the sample patients. This is confirmed by the significant statistical correlation between the amplitude of the SNAP and the reduced myelinated fibers observed. We used their motor trunk findings for that; however, it is known that leprosy affects nerve in asymmetric way, with some nerve fibers and regions being more affected than other what could represent a confounding factor [11].

At the moment of diagnosis, our patients frequently showed ulnar mNCS demyelinating pattern. In most PNL patients, the clinical evolution of leprosy neuropathy is notoriously slow and silent, making it a challenge to precisely determine the beginning of the pathological process and its neurophysiological expression. The findings of the nerve conduction studies evolution in leprosy neuropathy have been widely discussed in the literature. While some authors have described an axonal pattern in NCS [3-4,6,13], other authors have demonstrated demyelinating patterns [8-9].

Jardim et al. [7] also reported that, at diagnosis, leprosy patients had more demyelinating features than those who completed multidrug therapy. The same author demonstrated that the type of lesion found depends on the moment (early or late in disease evolution) the examination is performed [7]. 
Rambukkana et al. [1] had already described that demyelination and axonal damage may occur early in the $M$. leprae infection [1]. In fact, the both axonal damage and demyelinating features are present in leprosy neuropathy in different degree.

It was already described that PNL has a unique presentation of fibrosis, as it has a high degree of collagen deposit density that is not observed in other neuropathies [11]. In our sample, fibrosis was associated with axonal degeneration and it was described by Antunes et al. [14] that severe fibrosis is a frequent event in late-stage leprosy neuropathy when it causes irreversible functional damage [14]. This could indicate that the patients that do not have signs of axonal damage in their NCS may be in an earlier moment of the disease, when fibrosis is not present, as only the demyelinating features were observed in the NCS of these patients.

Overall, our data may suggest that demyelination may be present in leprosy neuropathy at the moment of the diagnosis and may be directly related to the duration of the neuropathy. Therefore, the diagnosis of PNL should be suspected in patients with the proper clinical context and with demyelinating features in the NCS, specially in locations where leprosy is endemic.

\section{References}

[1] Rambukkana A. Mycobacterium leprae-induced demyelination: A model for early nerve degeneration. Curr Opin Immunol. 2004;16(4):511-8. doi: 10.1016/j.coi.2004.05.021.

[2] Ridley DS, Jopling WH. Classification of leprosy according to immunity. A five-group system. Int J Lepr Other Mycobact Dis. 1966;34(3):255-73.

[3] Jardim MR, Antunes SLG, Santos AR, Nascimento OJM, Nery JAC, Sales $\mathrm{AM}$, et al. Criteria for diagnosis of pure neural leprosy. J Neurol. 2003;250(7):806-9. doi: 10.1007/s00415003-1081-5.

[4] Vital RT, Illarramendi $X$, Nascimento O, Hacker MA, Sarno EN, Jardim MR. Progression of leprosy neuropathy: A case series study. Brain Behav. 2012;2(3):249-55. doi: 10.1002/brb3.40.

[5] Griffin JW, Hsieh ST, McArthur JC, Cornblath DR. Laboratory testing in peripheral nerve disease. Neurol Clin. 1996;14(1):119-33. doi: 10.1016/s07338619(05)70246-2.

[6] Capadia GD, Shetty VP, Khambati FA, Ghate SD. Effect of corticosteroid usage combined with multidrug therapy on nerve damage assessed using nerve conduction studies: A prospective cohort study of 
365 untreated multibacillary leprosy patients. J Clin Neurophysiol. 2010;27(1):38-47. doi: 10.1097/WNP.0b013e3181cb426d.

[7] Jardim MR, Vital R, Hacker MA, Nascimento M, Balassiano SL, Sarno EN, et al. Leprosy neuropathy evaluated by NCS is independent of the patient's infectious state. Clin Neurol Neurosurg [Internet]. 2015; 131:5-10. doi: 10.1016/j.clineuro.2015.01.008.

[8] Rodriguez G, Pinto R, Gomez Y, Rengifo ML, Estrada OL, Sarmiento M, et al. Pure neuritic leprosy in patients from a high endemic region of Colombia. Lepr Rev. 2013;84(1):41-50.

[9] Jardim MR, Illarramendi X, Nascimento OJM, Nery JAC, Sales AM, Sampaio EP, et al. Pure neural leprosy: Steroids prevent neuropathy progression. Arq Neuropsiquiatr. 2007;65(4 A):969-73. doi: 10.1590/s0004$282 \times 2007000600009$.

[10] Kumar B, Kaur I, Dogra S, Kumaran MS. Pure neuritic leprosy in India: An appraisal. Int J Lepr Other Mycobact Dis. 2004;72(3):284-90. doi: 10.1489/0020-7349(2004)72< 284: PNLIIA $>2.0 . \mathrm{CO} ; 2$.

[11] Antunes SLG, Chimelli L, Jardim MR et al. Histopathological examination of nerve samples from pure neural leprosy patients: obtaining maximum information to improve diagnostic efficiency. Mem Inst Oswaldo Cruz
2012; 107 (2):246-53. doi: 10.1590/s007402762012000200015.

[12] Da Cunha FMB, Werneck MCM, Scola RH, Werneck LC. Pure neural leprosy: Diagnostic value of the polymerase chain reaction. Muscle and Nerve. 2006;33(3):409-14. doi: 10.1002/mus.20465.

[13] Jardim MR, Chimelli L, CorteReal $S$ et al. Clinical, electroneuromyographic and morphological studies of pure neural leprosy in a Brazilian referral centre. Leprosy Review 2004; 75:242-53.

[14] Antunes S, Jardim MR, Vital RT, Pascarelli BM, Nery JA, Amadeu TP, et al. Fibrosis: a distinguishing feature in the pathology of neural leprosy. Mem Inst Oswaldo Cruz. 2019;114(6):1-8. doi: 10.1590/0074-02760190056.

Conflict of interest: The authors declared no conflicts of interest.

Funding: The author Izabela Jardim Rodrigues Pitta received support from Fiotec (Fundação de Apoio a Fiocruz).

How to cite this article: Pitta IJR, Mietto BS, Camilo AS, Antunes SLG, Sarno EN, Jardim MR. Clinical, Neurophysiological and Histopathological correlations in Pure Neural Leprosy. Brazilian Journal of Case Reports. 2021 Oct-Dec;01(4):105113. 